University of Wollongong

Research Online

Faculty of Engineering and Information

Faculty of Engineering and Information

Sciences - Papers: Part A

Sciences

$1-1-2015$

\title{
Micro sheet hydroforming process of ultra-thin pure titanium foil
}

Hideki Sato

Tokyo Metropolitan University

Kenichi Manabe

Tokyo Metropolitan University

Dongbin Wei

University of Technology, Sydney, dwei@uow.edu.au

Zhengyi Jiang

University of Wollongong, jiang@uow.edu.au

Daiki Kondo

Tokyo Metropolitan University

Follow this and additional works at: https://ro.uow.edu.au/eispapers

Part of the Engineering Commons, and the Science and Technology Studies Commons

Research Online is the open access institutional repository for the University of Wollongong. For further information contact the UOW Library: research-pubs@uow.edu.au 


\title{
Micro sheet hydroforming process of ultra-thin pure titanium foil
}

\author{
Abstract \\ A micro hydromechanical deep drawing is carried out using the pure titanium and the effect of fluid \\ pressure on formability of pure titanium is investigated. The experiments are performed using the two \\ kinds of pure titanium foils (TR270C-H and TR270C-0) and stainless steel foil (SUS304-H) with 50 \\ thickness and the cylindrical and conical punches. As a result, it is found that the peeling off the oxide \\ film of pure titanium can be reduced by applying the fluid pressure because the friction force and contact \\ pressure between the blank and die decreases. However, the formability is lower for pure titanium than \\ that for stainless steel because the tensile strength is low and the friction force is easy to increase as the \\ friction force increases. In contrast, due to the low young modulus of pure titanium, the restriction of \\ wrinkling, decrease of friction force and friction holding effect can be obtained at low fluid pressure.

\section{Keywords} \\ foil, titanium, pure, thin, ultra, process, hydroforming, sheet, micro \\ Disciplines \\ Engineering | Science and Technology Studies

\section{Publication Details} \\ Sato, H., Manabe, K., Wei, D., Jiang, Z. \& Kondo, D. (2015). Micro sheet hydroforming process of ultra-thin \\ pure titanium foil. Key Engineering Materials, 626 397-401.
}




\title{
Micro Sheet Hydroforming Process of Ultra-Thin Pure Titanium Foil
}

\author{
Hideki Sato ${ }^{1, a^{*}}$, Ken-ichi Manabe ${ }^{1, b}$, Dongbin $\mathrm{Wei}^{2, \mathrm{c}}$, Zhengyi Jiang ${ }^{3, \mathrm{~d}}$ \\ and Daiki Kondo ${ }^{1, e}$ \\ ${ }^{1}$ Tokyo Metropolitan University, 1-1 Minamiosawa, Hachioji, Tokyo 192-0397, Japan \\ ${ }^{2}$ University of Technology, Sydney, 15, Broadway Ultimo, NSW, 2007, Australia \\ ${ }^{3}$ University of Wollongong, Northfields, Avenue, Wollongong, NSW, 2552, Australia \\ a sato-hideki@ed.tmu.ac.jp, ${ }^{\mathrm{b}}$ manabe@tmu.ac.jp, 'Dongbin.Wei@uts.edu.au, ${ }^{\mathrm{c}}$ jiang@uow.edu.au, \\ ekondo-daiki@ed.tmu.ac.jp
}

Keywords: Micro sheet hydroforming, Micro hydromechanical deep drawing, Pure titanium, Fluid pressure.

\begin{abstract}
A micro hydromechanical deep drawing is carried out using the pure titanium and the effect of fluid pressure on formability of pure titanium is investigated. The experiments are performed using the two kinds of pure titanium foils (TR270C-H and TR270C-O) and stainless steel foil (SUS304-H) with 50 thickness and the cylindrical and conical punches. As a result, it is found that the peeling off the oxide film of pure titanium can be reduced by applying the fluid pressure because the friction force and contact pressure between the blank and die decreases. However, the formability is lower for pure titanium than that for stainless steel because the tensile strength is low and the friction force is easy to increase as the friction force increases. In contrast, due to the low young modulus of pure titanium, the restriction of wrinkling, decrease of friction force and friction holding effect can be obtained at low fluid pressure.
\end{abstract}

\section{Introduction}

By ongoing the miniaturization of modern devices, the demand of micro products is growing in many industries. In particular, a pure titanium is today paid attention in the medical and chemical field due to its properties of excellent corrosion resistance and biocompatibility [1]. The pure titanium is known as a material that has the high affinity and easily occurs the adhesion and seizure when its oxide film is broken during the sheet forming process. In general, the lubrication and surface treatment of tools have been employed in the forming of pure titanium in order to avoid the adhesion and seizure. However, with scaling down to micro scale, the friction force increases due to the lubricant cannot be kept [2]. Moreover, even though the anodized pure titanium foil is used, the generated wear debris scratches the surface during the ironing process because it is much larger than the substrate of pure titanium [3]. It finally causes the adhesion and seizure of pure titanium in micro sheet forming. In addition, although the pure titanium has the excellent mechanical properties in macro forming, it might deteriorate with scaling down to micro scale due to the grain size effect. It is because the ductility and tensile strength decreases because the number of grain size along the thickness decreases [4]. These high friction force and poor mechanical properties generally result the low drawability in micro sheet forming [5].

Sheet hydroforming is a forming method using the fluid pressure instead of the die. It is reported that the macro sheet hydroforming can reduce the friction force due to the hydrodynamic lubrication [6]. Therefore, it is expected that the fluid pressure can reduce the peeling off the oxide film and restrain the adhesion and seizure of pure titanium. Moreover, the drawability can be improved due to several effects of fluid pressure in hydromechanical deep drawing, such as the friction holding effect and hydrodynamic lubrication effect [7]. Previously, the authors have developed a micro hydromechanical deep drawing (MHDD) to utilize these advantages in micro sheet forming [8]. In this study, the micro hydromechanical deep drawing (MHDD) is carried out using the pure titanium to investigate the effect of fluid pressure on frictional behavior and drawability of pure titanium. 


\section{Experimental}

Experimental procedure. To improve the handing and positioning of tiny workpiece, the blanking and drawing tooling set developed by Authors was used as shown in Fig. 1 [8]. The pressure generation, blanking, drawing and knockout processes can be performed in the same axis in this machine. A pump with maximum fluid pressure of 20MPa was used. The materials used are the pure titanium foil (TR270C-H) and the stainless steel (SUS304-H) with 50 $\mu \mathrm{m}$ thickness as the comparison material. The mechanical properties of pure titanium foils are listed in Table 1 . The experiment was performed using the cylindrical punch. The tool dimensions are shown in Fig. 2(a). The constant gap method was adopted for the blank holder. The drawing ratio is $\mathrm{DR}\left(=D_{\mathrm{b}} / D_{\mathrm{p}}\right)=1.74$. The dry friction was employed in MDD and the machine oil was used in MHDD. The punch speed was set up at $0.4 \mathrm{~mm} / \mathrm{s}$.

In other to investigate the friction holding effect, the experiment was also carried out using conical punch in which it is easy to observe the shape accuracy at side wall. As for the conical MHDD, the pure titanium foil (TR270C-O) was used. The mechanical properties and the tool dimension are listed in Table 1 and shown in Fig. 2(b). The drawing ratio is $\mathrm{DR}\left(=D_{\mathrm{b}} / D_{\mathrm{lp}}\right)=2.20$.

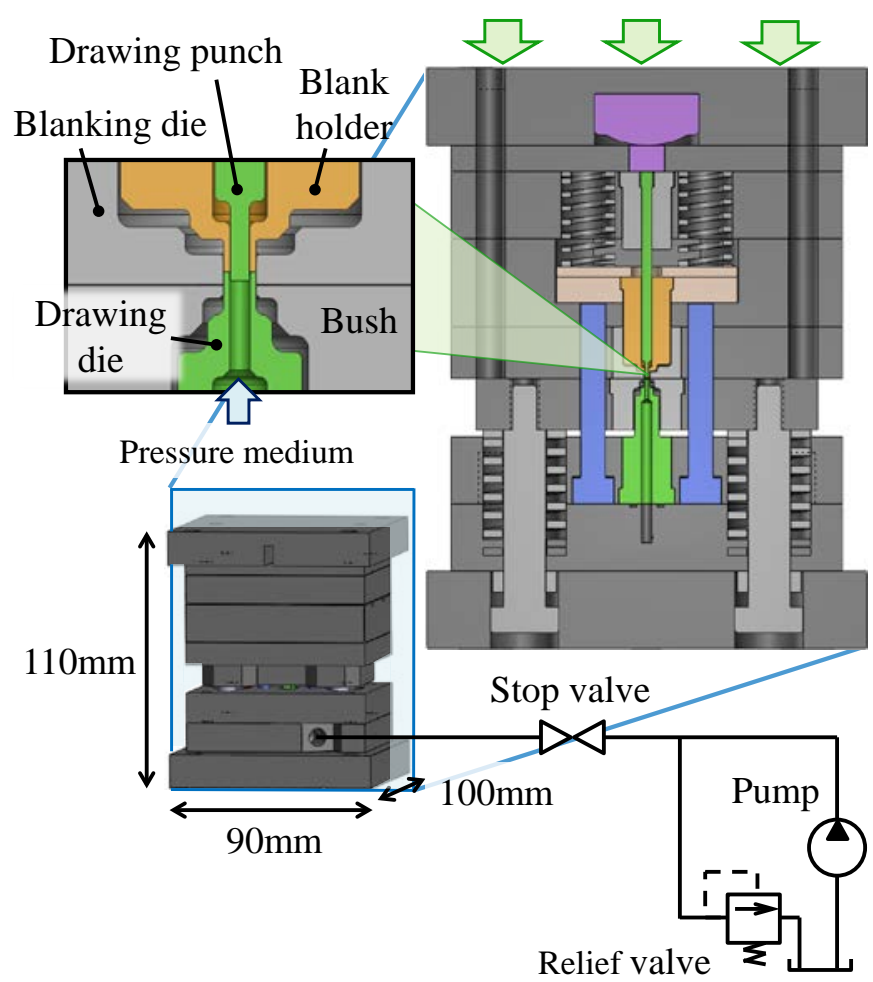

Figure 1 Schematic of MHDD tools set and hydraulic system.

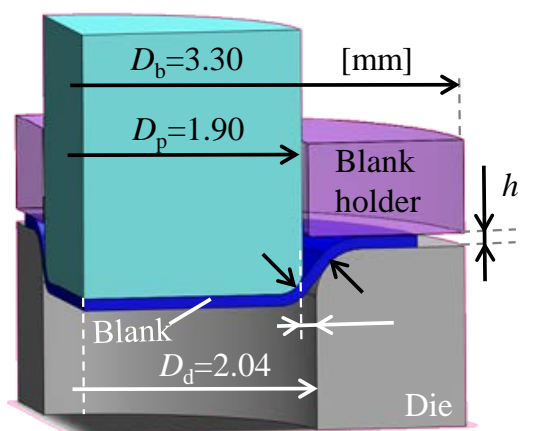

(a) Cylindrical punch

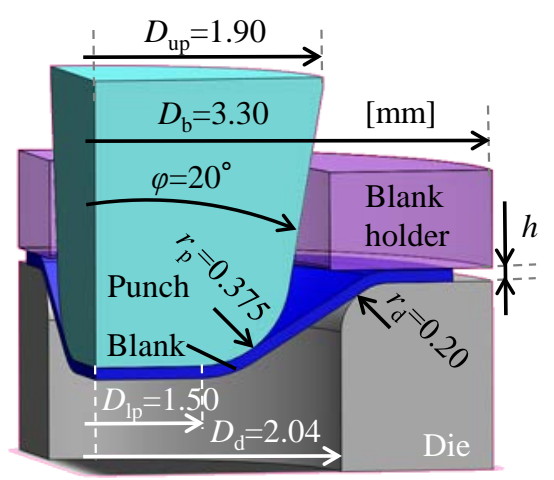

(b) Conical punch

Figure 2 Tooling dimensions of cylindrical and conical punches at MDD and MHDD.

Table 1 Mechanical properties of pure titanium and stainless steel foils

\begin{tabular}{|c|c|c|c|c|}
\hline Materials & $\begin{array}{c}\text { Young's modulus } \\
E[\mathrm{GPa}]\end{array}$ & $\begin{array}{c}\text { Yield stress } \\
\sigma_{y}[\mathrm{MPa}]\end{array}$ & $\begin{array}{c}\text { Tensile strength } \\
\sigma_{B}[\mathrm{MPa}]\end{array}$ & $\begin{array}{c}\text { Elongation } \\
l[\%]\end{array}$ \\
\hline TR270C-H & 117 & 601 & 807 & 1.4 \\
\hline SUS304-H & 193 & 1217 & 1331 & 2.4 \\
\hline TR270C-O & 93 & 294 & 446 & 13.8 \\
\hline
\end{tabular}


Effective punch force. A measured punch force $F_{H}$ was recalculated by the effective punch force $F_{\mathrm{E}}$ to evaluate the friction force in MDD and MHDD. The effective punch force is calculated by the measured punch force subtracted from the force to push the punch by the fluid pressure $F_{\mathrm{P}}$. It is expressed by

$P_{\mathrm{E}}=P_{\mathrm{H}}-F_{\mathrm{H}}=F_{\mathrm{S}}+F_{\mathrm{B}}+F_{\mathrm{F}}$

where $F_{\mathrm{S}}$ is the pure drawing force, $F_{\mathrm{B}}$ is the bending force and $F_{\mathrm{F}}$ is the friction force. The pure drawing force and bending force is almost the same at the same material and tool dimension. Therefore, the friction force can be evaluated by the difference of effective punch force in each condition.

\section{Results and discussion}

Frictional behavior in cylindrical MHDD of pure titanium. Fig. 3 shows the normalized effective punch force at cylindrical MDD and MHDD. The difference of effective punch force can be evaluated the friction force at each condition. By applying the fluid pressure in MHDD, the friction force is once reduced and becomes high at high fluid pressure. Therefore, the friction force can be reduced by applying the appropriate fluid pressure in MHDD. This decrease of friction force in MHDD means that the fluid pressure pushes up the blank and reduces the contact pressure between the blank and die. Accordingly, the damage of oxide file of pure titanium can be reduced due to the low contact pressure between the blank and die in MHDD. Moreover, by increasing the fluid pressure more, the blank does not contact with the die directly because the hydrodynamic lubrication is obtained. Therefore, it is expected that the adhesion and seizure of pure titanium can be prevented in MHDD.

Additionally, the required fluid pressure to reduce the friction force is smaller for the pure titanium than that for the stainless steel as shown in Fig. 3. As for the pure titanium, the yield stress is smaller than that of stainless steel. It means that the contact pressure between the blank and die is low for the pure titanium. Therefore, the fluid medium leaks between them at the low fluid pressure and it is easy to obtain the hydrodynamic lubrication. In contrast, the friction force increases from almost the same relative fluid pressure to yield stress $p / \sigma_{\mathrm{y}}$ in both materials. In other word, the friction force increases even at the low fluid pressure in pure titanium because the yield stress of pure titanium is lower than that of stainless steel. In pure titanium, the young modulus is low and it causes the low stiffness of blank. Therefore, the blank is easily deformed and the force pushing the blank by the fluid pressure is easily transmitted to the blank holder. It causes the high friction force between the blank and blank holder. It was found that the effect of fluid pressure on friction force is more sensitive for the pure titanium than that for stainless steel due to its low yield stress and young modulus.

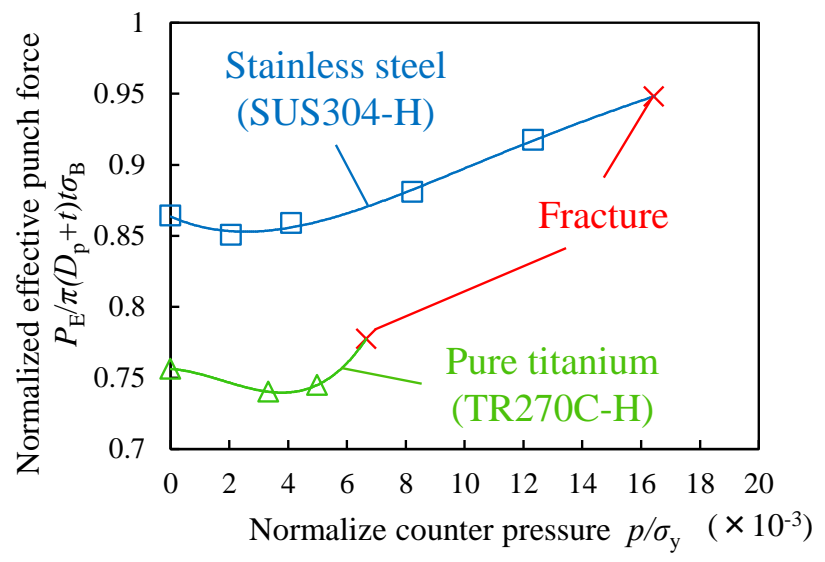

Figure 3 Effect of fluid pressure on change of friction force in cylindrical MHDD of pure titanium.

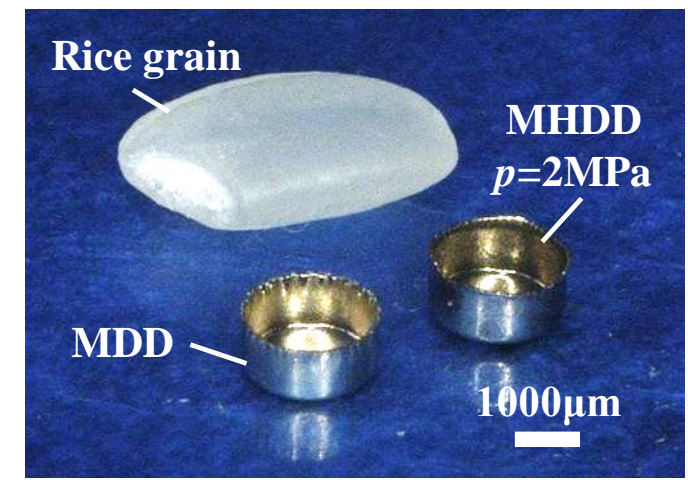

Figure 4 Appearance of micro cups of pure titanium (TR270C-H) on ruler drawn by cylindrical MDD and MHDD. 
Formability of pure titanium in cylindrical MHDD. Fig. 4 shows the micro cups drawn by the cylindrical punch. The pure titanium micro cups were successfully fabricated at MHDD although the punch shoulder radius is small. The drawn micro cups are smaller than the rice grain. Fig. 5 shows the winkling and fracture of cups drawn by MDD and MHDD of pure titanium. The occurrence of winkling is restricted by applying the fluid pressure of $2 \mathrm{MPa}$. In contrast, the fracture finally occurs at the fluid pressure of 4MPa. As the fluid pressure increases, the wrinkling decreases because the fluid pressure plays a role of blank holder stress in MHDD. However, with increasing fluid pressure more, the fracture occurs at punch shoulder due to the increase of friction force as shown in Fig. 3.

Fig. 6 shows the effect of fluid pressure on wrinkling and fracture of micro cups. In both of materials, the required relative fluid pressure to young modulus $p / E$ to prevent the wrinkling is the same. It means that the required fluid pressure is low for the pure titanium because its young modulus is lower than that of stainless steel. On the other hand, the fracture occurs before the success zone appears in the pure titanium although there is a large success zone in the stainless steel. As shown in Fig. 3, the friction force easily increases for the pure titanium foil even in the low fluid pressure due to the low young modulus. Moreover, the tensile strength of pure titanium is lower than that of stainless steel. It means that the allowable load is low for pure titanium. These are reasons why the fracture occurs at low fluid pressure and there is not the success zone in the case of pure titanium. The increase of friction force can be improved by the radial pressure. The radial pressure easily lubricates not only between the blank and die, but also between the blank and blank holder because it is applied from the blank edge directly. Moreover, the compression force is applied at the blank edge by the radial pressure which reduces the loading force of blank. Thus, it is expected that the improvement of friction force and formability can be obtained by applying the radial pressure.

Friction holding effect of conical cups in MHDD. The formability can be further improved by the friction holding effect at MHDD in which the friction force between the blank and punch reduces the loading force of blank at punch shoulder. The friction force can be evaluated by the straightness at side wall because the friction holding effect is obtained when the straightness at side wall becomes zero. Fig. 7 shows the effect of fluid pressure on straightness at conical micro cups. In MDD, the straightness is low. In contrast, the straightness becomes high in MHDD and increases as the fluid pressure increases. Because the low young modulus in pure titanium causes the low stiffness, the blank is easy to deform. It means that the required fluid pressure is low to obtain the high straightness. It was found that the fiction holding effect can be obtained at low fluid pressure in the pure titanium foil. Moreover, to obtain the high shape accuracy in conventional deep drawing, the drawn products have to be contacted with the die and the shape of die is transcribed to the products. However, due to the high contact pressure during this process, it is easy to peel off the oxide film and cause the adhesion and seizure in pure titanium. On the other hand, by applying the fluid pressure, the high shape accuracy was obtained without the contact with the die in MHDD.

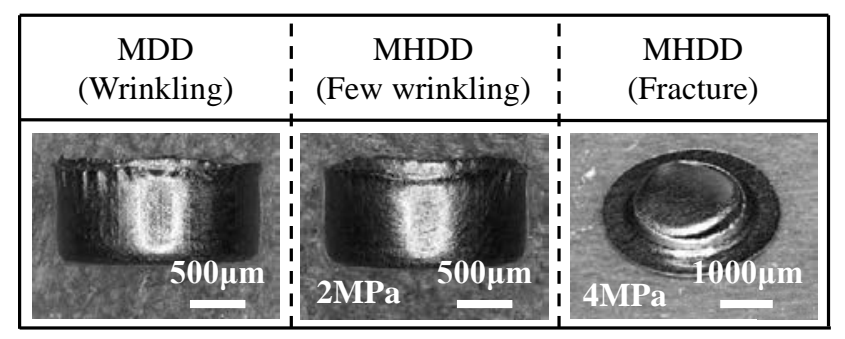

Figure 5 Photos of wrinkling and fracture cups in MHDD of pure titanium (TR270C-H).

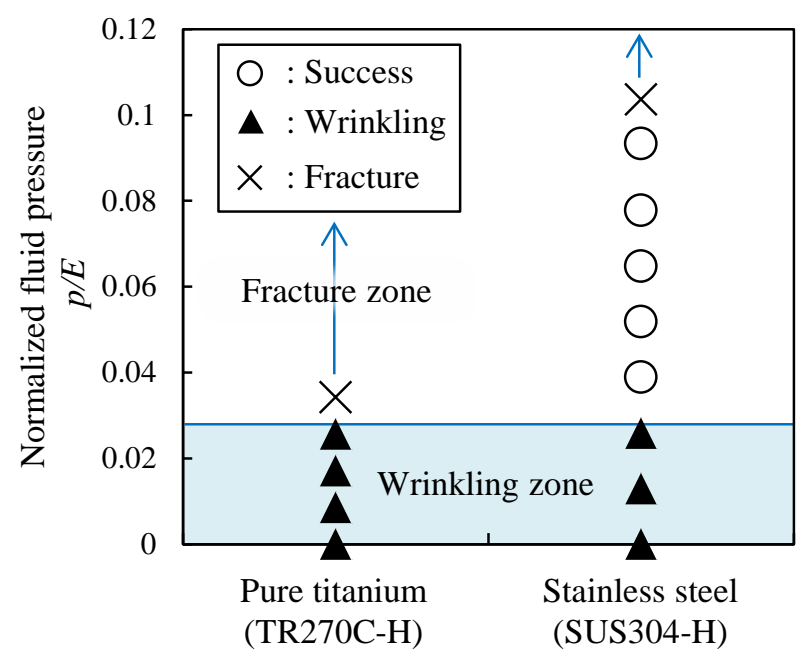

Figure 6 Effect of normalized fluid pressure on wrinkling and fractuer in MHDD. 

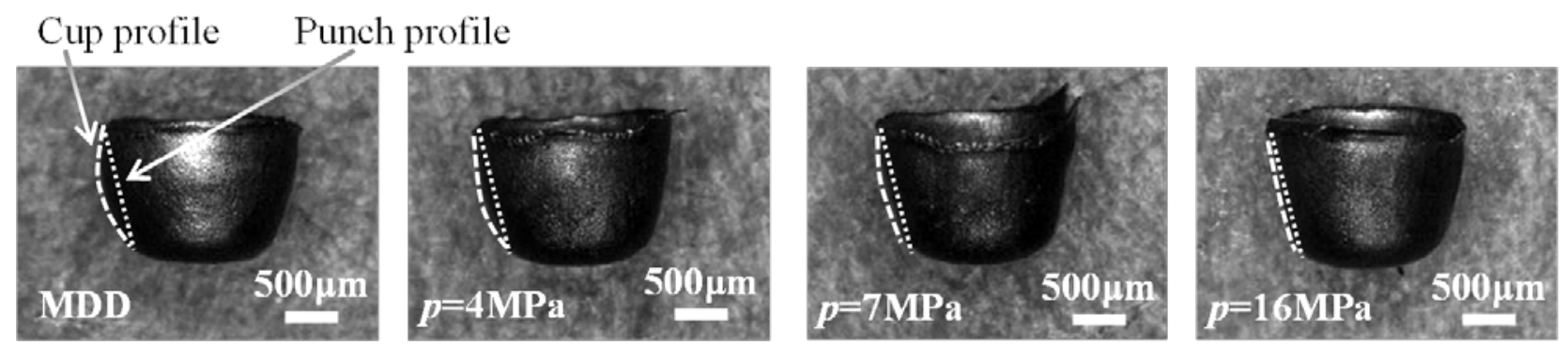

Figure 7 Effect of fluid pressure on straightness at conical micro cups of pure titanium (TR270C-O).

\section{Conclusion}

The micro hydromechanical deep drawing was carried out using the pure titanium in this study to investigate the effect of fluid pressure on frictional behavior and drawability of pure titanium. The experiments were performed using the pure titanium foils (TR270C-H and TR270C-O) and the stainless steel foil of $50 \mu \mathrm{m}$ in thickness as the comparison material. In MHDD, the friction force can be reduced by applying fluid pressure. It shows that the peeling off the oxide film of pure titanium decreases because the contact pressure between the blank and die is reduced. However, the formability is lower for pure titanium than that for stainless steel. It is because the tensile strength is low and the friction force easily increases with the increase of fluid pressure in pure titanium. In contrast, it was clarified that the winkling can be avoided and the friction holding effect can be obtained by applying the fluid pressure in MHDD. The required fluid pressure to obtain the decrease of friction force, restriction of wrinkling and friction holding effect is lower for the pure titanium than that for the stainless steel. It seems to be because the low young modulus in the pure titanium causes the low stiffness and makes it easy to deform the blank.

\section{References}

[1] K. Wang, The Use of Titanium for Medical Applications in the USA, Materials Science and Engineering, 213, (1996), 134-137.

[2] K.V. Putten, M. Franzke, G. Hirt, Size Effect on Friction and Yielding in Wire Flat Rolling, Proceedings of the 2nd International Conference on New Forming Technology, (2007), 583-592.

[3] T. Shimizu, S. Iwaoka, Ken-ichi Manabe, Y. Teranishi, K. Morikawa, Tribological Properties of Anodized Pure Titanium Foils in Micro-Deep Drawing with Ironing, Proceedings of the $15^{\text {th }}$ International Conference on Advances in Materials \& Processing Technologies, (2012), 11790.

[4] M. W. Fu, W. L. Chan, Geometry and Grain Size Effects on the Fracture Behavior of Sheet Metal in Micro-Scale Plastic Deformation, Materials and Design, 32, (2011), 4738-4746.

[5] F. Vollertsen, Effects on the Deep Drawing Diagram in Micro Forming, Production Engineering, 6, (2012), 11-18.

[6] K. Nakamura, T. Nakagawa, Sheet Metal Forming with Hydraulic Counter Pressure in Japan, Annals of the CIRP, 36, (1987), 191-194.

[7] K. Nakamura, T. Nakagawa, Fracture Mechanism and Fracture Control in Deep Drawing with Hydraulic Counter Pressure - Studies on Hydraulic Counter Pressure Forming I, Journal of the Japan Society for Technology of Plasticity (in Japanese), 25, (1984), 831-838.

[8] H. Sato, K. Manabe, K. Ito, D. Wei, Z. Jiang, Development of Servo-Type Micro-Hydromechanical Deep-Drawing Apparatus and Micro Deep-Drawing Experiments of Circular Cups, Journal of the Japan Society for Technology of Plasticity (in Japanese), 55, (2014), 44-49. 